\title{
Observations of Complex Molecules in Low-Mass Protostars
}

\author{
Nami Sakai and Satoshi Yamamoto \\ Department of Physics and Research Center for the Early Universe, The University of Tokyo, \\ Bunkyo-ku, Tokyo 113-0033, Japan \\ email: nami@phys.s.u-tokyo.ac.jp
}

\begin{abstract}
Low-mass star forming regions are rich inventories of complex organic molecules. Furthermore, they show significant chemical diversity even among sources in a similar physical evolutionary stage (i.e. Class 0 sources). One distinct case is the hot corino chemistry characterized by rich existence of saturated complex organic molecules such as $\mathrm{HCOOCH}_{3}$ and $\mathrm{C}_{2} \mathrm{H}_{5} \mathrm{CN}$, whereas the other is the warm carbon-chain chemistry (WCCC) characterized by extraordinary richness of unsaturated complex organic molecules such as carbon-chain molecules. We here summarize these observational achievements during the last decade, and present a unified picture of carbon chemistry in low-mass protostellar cores. The chemical diversity most likely originates from the source-to-source difference in chemical compositions of grain mantles. In particular, the gas-phase abundance of $\mathrm{CH}_{4}$ evaporated from grain mantles is thought to be a key factor for appearance of WCCC. The origin of the diversity and its evolution to protopranetary disks are discussed.
\end{abstract}

Keywords. ISM: molecules, ISM: abundances, stars: formation

\section{Introduction}

So far more than 160 interstellar molecules have been identified mainly by radioastronomical observations, and about $1 / 3$ of them are 'complex' molecules having 6 atoms or more (c.f. Herbst \& van Dishoeck 2009). This clearly indicates the high chemical complexity of interstellar medium, which would ultimately be related to an origin of rich substances in our solar system. Hence, a detailed understanding of the chemical composition and its evolution in star forming regions is a central issue for astrochemistry.

Complex organic molecules (COMs) are classified into two general groups, saturated COMs and unsaturated COMs. Saturated COMs are fully or almost fully hydrogenated molecules such as $\mathrm{CH}_{3} \mathrm{OH}, \mathrm{HCOOCH}_{3},\left(\mathrm{CH}_{3}\right)_{2} \mathrm{O}$, and $\mathrm{C}_{2} \mathrm{H}_{5} \mathrm{CN}$. These molecules are known to exist abundantly in hot cores in high-mass star forming regions. Since the gas-phase chemical reactions cannot produce them efficiently, they are thought to be formed by grain surface reactions (e.g., Charnley et al. 1997; Watanabe \& Kouchi 2002; Garrod \& Herbst 2006; Herbst \& van Dishoeck 2009). The produced molecules stay on grain mantles in the cold phase, and they are librated into the gas phase after the birth of high-mass stars. Since 1980s, the hot core chemistry has extensively been studied to account for the observational results in various high-mass star forming regions and external galaxies (e.g., Blake et al. 1987; Millar et al. 1991; Nomura \& Millar 2004; Herbst \& van Dishoeck 2009). On the other hand, unsaturated COMs are represented by so called carbon-chain molecules having rich multiple bonds. They are known to exist abundantly and preferentially in young starless cores such as TMC-1. In fact, carbonchain molecules are dominant organic molecules in TMC-1, according to the spectral line survey by Kaifu et al. (2004). Carbon-chain molecules are thought to be produced in the gas phase starting from $\mathrm{C}^{+}$and $\mathrm{C}$, and hence, they are only abundant in young starless 
cores, where $\mathrm{C}^{+}$and $\mathrm{C}$ have not been converted to $\mathrm{CO}$ completely (Suzuki et al. 1992; Benson et al. 1998).

In contrast to the extensive astrochemical studies on high-mass star forming regions and cold starless cores, chemistry of low-mass star forming regions was sparsely studied until 1990s. A pioneering study toward this direction is the spectral line survey of the low-mass Class 0 protostar, IRAS 16293-2422, by Blake et al. (1994) and van Dishoeck et al. (1995). They studied the chemical composition of this source in an unbiased way for the first time. Although they found rich $\mathrm{CH}_{3} \mathrm{OH}$ and $\mathrm{SO}_{2}$, which can be recognized as a signature of the hot-core like chemistry, they could not detect the lines of larger COMs such as $\mathrm{HCOOCH}_{3},\left(\mathrm{CH}_{3}\right)_{2} \mathrm{O}$, and $\mathrm{C}_{2} \mathrm{H}_{5} \mathrm{CN}$ at that time, probably because of insufficient sensitivities in their observations. The chemical composition of low-mass star forming regions is of fundamental importance, because it can be regarded as the initial condition for chemical evolution to protoplanetary disks and planets. Thanks to great improvements of sensitivity in millimeter- and submillimeter-wave observations, such studies have extensively been carried out in 2000s, resulting in the following discoveries.

\section{Hot Corino Chemistry and Warm Carbon-Chain Chemistry}

First discovery was the detection of large saturated COMs such as $\mathrm{HCOOCH}_{3}$, $\left(\mathrm{CH}_{3}\right)_{2} \mathrm{O}$, and $\mathrm{C}_{2} \mathrm{H}_{5} \mathrm{CN}$ in the low-mass star-forming region, IRAS16293-2422 (Cazaux et al. 2003), revealing that the hot-core like chemistry is also occurring even in a low-mass source. Furthermore, the interferometric observations showed that these molecules are distributed within a few $100 \mathrm{AU}$ region (at temperature of $\sim 100 \mathrm{~K}$ ) around the protostar (Bottinelli et al. 2004b; Kuan et al. 2004). These results along with subsequent detections of the saturated COMs in other low-mass star forming regions clearly demonstrated that fairly complex organic molecules are already formed in the protostellar-core phase, which would be brought into protoplanetary disks. Then, it was thought that they may be related to an origin of pre-solar organic molecules found in meteorites (e.g., Cronin \& Pizzarello 1997 and references therein). Just as in the case of the hot core chemistry in high-mass star forming regions, saturated COMs are formed on dust grains during cold and warm-up phases (Garrod \& Herbst 2006), and are liberated into the gas phase in a small hot region (a few $100 \mathrm{AU}$ ) around the protostar, called hot corino. Now four Class 0 protostars (IRAS 16293-2422, NGC1333 IRAS4A, IRAS4B, and IRAS2A) are known to harbor hot corinos (Cazaux et al. 2003; Bottinelli et al. 2004a; Jørgensen et al. 2005; Sakai et al. 2006; Bottinelli et al. 2007).

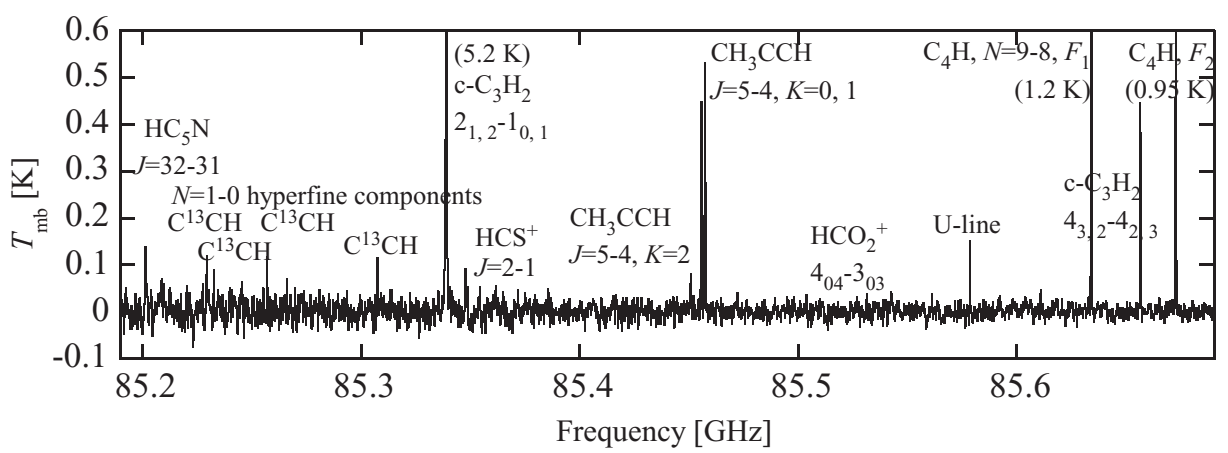

Figure 1. A portion of the spectral line survey toward L1527 observed with the Nobeyama 45 $\mathrm{m}$ telescope. Peak intensities are reduced by a factor of about 2 because of the narrow line width $\left(\sim 0.5 \mathrm{~km} \mathrm{~s}^{-1}\right)$ in comparison with the velocity resolution of the spectrometer $\left(\sim 0.8 \mathrm{~km} \mathrm{~s}^{-1}\right)$. In addition to the bright lines of $\mathrm{C}_{4} \mathrm{H}$ and $\mathrm{c}^{-} \mathrm{C}_{3} \mathrm{H}_{2}$, even the very high excitation line of $\mathrm{HC}_{5} \mathrm{~N}$ $\left(J=32-31, E_{\mathrm{u}}=67 \mathrm{~K}\right)$ can be seen. 
On the other hand, the extraordinary richness of unsaturated COMs such as $\mathrm{C}_{4} \mathrm{H}$, $\mathrm{C}_{4} \mathrm{H}_{2}$, and $\mathrm{HC}_{5} \mathrm{~N}$ was found in the lukewarm region around the low-mass Class 0 protostar, L1527 (Sakai et al. 2008a). For instance, the high excitation lines of $\mathrm{C}_{4} \mathrm{H}(N=9-8)$, $\mathrm{C}_{2} \mathrm{H}(N=3-2)$ were detected with very high intensities (Figure 1). Emissions of various long carbon-chain molecules and their anions like $\mathrm{C}_{4} \mathrm{H}^{-}$and $\mathrm{C}_{6} \mathrm{H}^{-}$were also detected (e.g. Sakai et al. 2007, 2008b; Agúndez et al. (2008)) (Figure 2), while the lines of saturated COMs were not (Sakai et al. 2008c). Hence, the protostellar core L1527 has a completely different chemical composition from that of hot corinos. A small mapping observation of $\mathrm{C}_{4} \mathrm{H}$ with the Nobeyama $45 \mathrm{~m}$ telescope shows that the distribution is concentrated toward the protostar in a few thousand AU. More importantly, the line width becomes broader toward the protostar position. It is therefore highly likely that carbon-chain molecules do exist even in the gas infalling to the protostar. This result is striking, since it had been thought that carbon-chain molecules are abundant only in young starless cores and deficient in the evolved starless cores and star forming cores (e.g., Suzuki et al. 1992; Benson et al. 1998). To account for these results, a new production scheme of carbon-chain molecules triggered by evaporation of $\mathrm{CH}_{4}$ from ice mantles was proposed (Sakai et al. 2008a). It is called warm carbon-chain chemistry (WCCC). The sublimation temperature of $\mathrm{CH}_{4}$ is $25 \mathrm{~K}$ (Collings et al. 2004), being much lower than

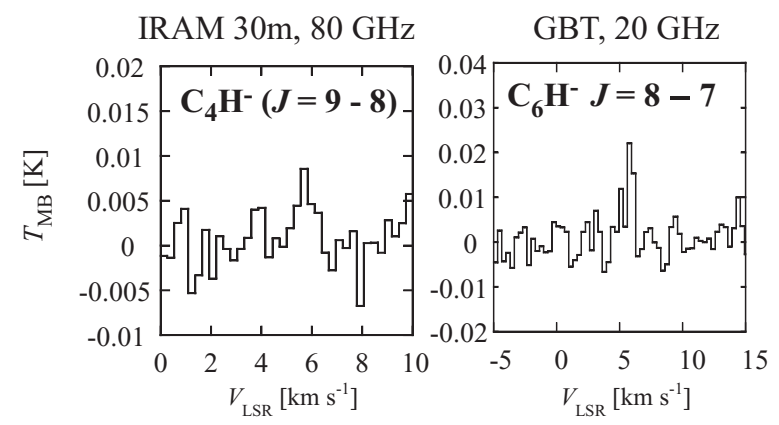

Figure 2. Spectral line profiles of carbon-chain anions observed toward L1527 (Sakai et al.
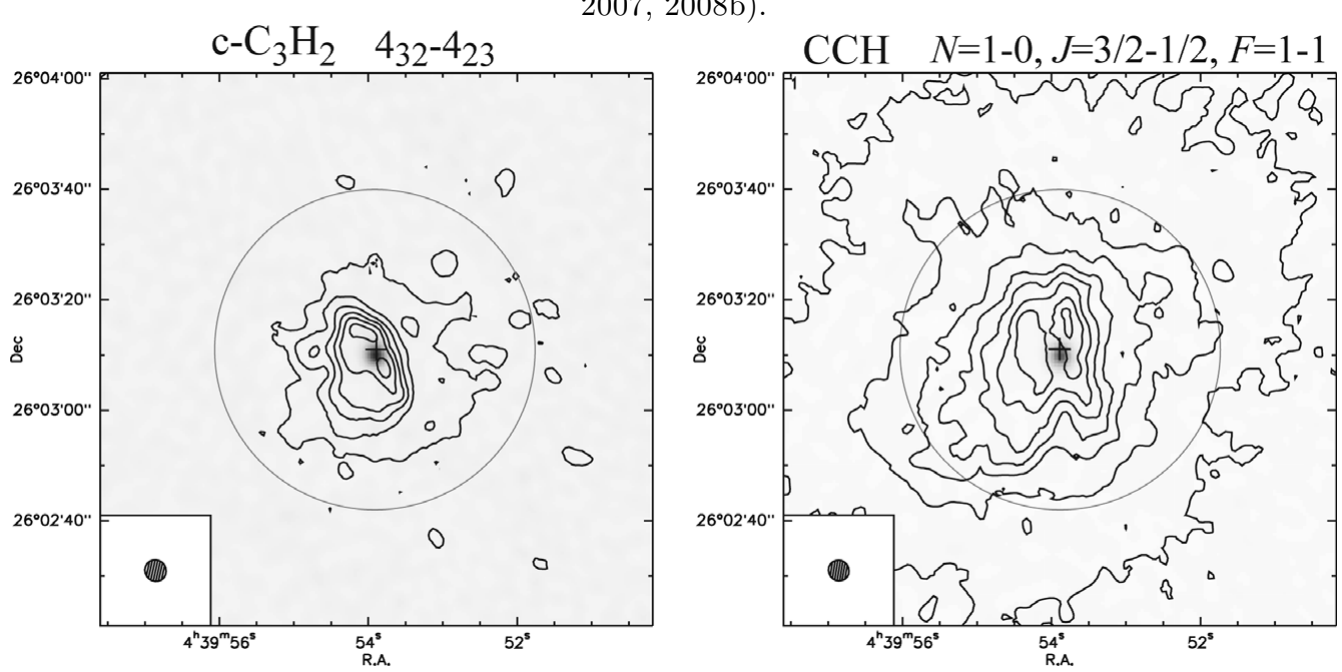

Figure 3. Integrated intensity maps of $\mathrm{c}_{-} \mathrm{C}_{3} \mathrm{H}_{2}$ and $\mathrm{CCH}$ observed toward L1527 with PdBI (Sakai et al. 2010. The maps are prepared by combining the single dish (IRAM $30 \mathrm{~m}$ ) data to recover the extended component. The circles represent a field of view of PdBI. 
that of $\mathrm{H}_{2} \mathrm{O}$. Hence, $\mathrm{CH}_{4}$ is evaporated in a warm region extending to a few thousand $\mathrm{AU}$, and reacts with $\mathrm{C}^{+}$in the gas phase to produce various carbon-chain molecules. The basic mechanism of WCCC was independently confirmed by chemical model calculations (Aikawa et al. 2008; Hassel et al. 2008; Harada \& Herbst 2008).

An important question is then whether such peculiar chemistry is specific to L1527 or not. With this in mind, we searched for a similar source among many low-mass protostars, and finally found the second WCCC source, IRAS 15398-3359, in Lupus molecular cloud 1 ( $d=150$ pc) (Sakai et al. 2009a). Its spectral features resemble those in L1527, and various carbon-chain molecules are as abundant as in L1527. Furthermore, the distribution of the high excitation line of $\mathrm{CCH}(N=4-3)$ shows central concentration around the protostar. According to the Spitzer observations, a deep infrared absorption feature of solid $\mathrm{CH}_{4}$ is seen toward this source (Öberg et al. 2008), implying a favorable condition for WCCC. The discovery of the WCCC source outside the Taurus region suggests that WCCC can no longer be ascribed to a peculiarity of a particular region, but should be regarded as one of characteristic chemical features of low-mass protostellar cores.

Recently, we conducted an interferometric observation of carbon-chain molecules and their related molecules toward L1527 with PdBI, and confirmed that carbon-chain molecules are distributed within a few thousand AU around the protostar, as shown in Figure 3 (Sakai et al. 2010). The distributions show a steep increase inward of a radius of 500-1000 AU from the protostar, implying an abundance jump by an order of magnitude. The radius corresponds to the dust temperature of $20-30 \mathrm{~K}$, which is just around the evaporation temperature of $\mathrm{CH}_{4}$. This result further supports the WCCC in L1527. More interestingly, a small dip (500 AU scale) can be seen toward the protostar position. We carried out a simple radiative transfer calculation by employing the density and temperature structure of the L1527 protostellar core reported by Jørgensen et al. (2002), and found that the abundance of the carbon-chain molecules decreases in the innermost part by a factor of 10 . Nevertheless, carbon-chain molecules are thought to survive partly in the gas infalling to the protostar, since the blue- and red-shifted components by $\sim 1 \mathrm{~km} \mathrm{~s}^{-1}$ are centrally concentrated (See Figures 2-4 of Sakai et al. (2008a)). Hence, it is likely that a portion of carbon-chain molecules produced in the Class 0 phase will be delivered to the protostellar disk.

\section{Origin and Future of the Chemical Diversity}

So far, it has been thought that the chemical compositions of low-mass star forming regions are more or less uniform, and hence, the chemical evolution from a protostellar disk to a protoplanetary disk has no significant variations. However, the above observational
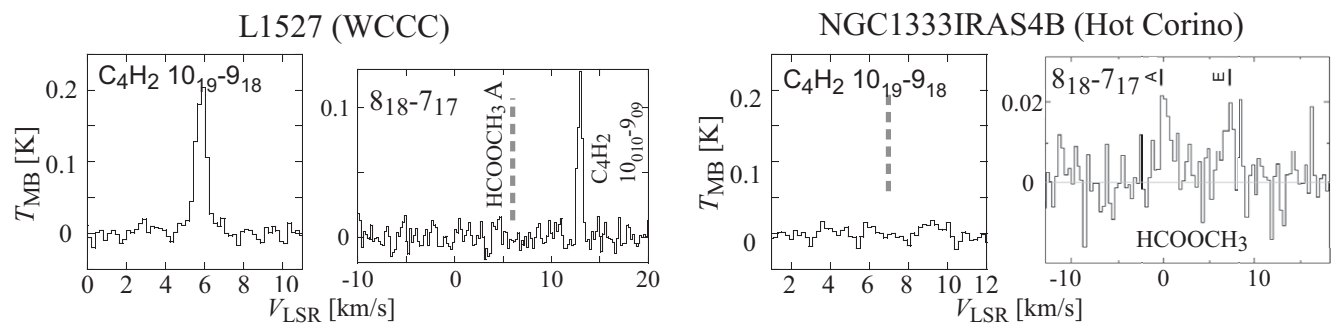

Figure 4. Chemical diversity of the Class 0 sources. In the WCCC source L1527, the carbon-chain molecules are abundant, whereas the complex organic molecules tend to be deficient. In the hot corino source NGC1333 IRAS4B, the situation is opposite. The $\mathrm{C}_{4} \mathrm{H}_{2}$ and $\mathrm{HCOOCH}_{3}$ spectra in L1527 are taken from Sakai et al. (2008a) and Sakai et al. (2008c), respectively. The $\mathrm{HCOOCH}_{3}$ spectrum in NGC1333 IRAS4B is from Sakai et al. (2006). 
results clearly demonstrate diversity of chemical compositions in low-mass protostellar cores. At present, we know the hot corino and WCCC sources as the two extreme cases. These two types of sources show exclusive nature in chemical compositions. In hot corinos, unsaturated COMs such as carbon-chain molecules are generally deficient, whereas in WCCC sources, saturated COMS such as $\mathrm{HCOOCH}_{3}$ are deficient (Figure 4). This implies that the chemical evolution to the protoplanetary disk is not a single pathway, and the chemical compositions of protoplanetary disks could be different from source to source.

\subsection{Carbon Chemistry around Low-Mass Protostar}

To consider the origin of the chemical diversity, we first summarize the chemical processes which would occur during the temperature raise due to protostellar activities. We start from a cold $(T<10 \mathrm{~K})$ dense starless core, where most of the molecules except for $\mathrm{H}_{2}$ are depleted on dust grains and no carbon chemistry is occurring. After the birth of the protostar, the temperature starts to rise. We can define the following three major steps in carbon-chemistry.

(1) Restart of carbon-chemistry $(T \sim 20 \mathrm{~K})$ : At about $20 \mathrm{~K}$, CO is evaporated from grain mantles, which restarts the basic carbon-chemistry by the $\mathrm{He}^{+}+\mathrm{CO}->\mathrm{C}^{+}+\mathrm{O}+\mathrm{He}$ reaction. The produced $\mathrm{C}^{+}$reacts with $\mathrm{H}_{2}$ to form $\mathrm{CH}_{2}^{+}$, and then makes various organic molecules. This is essentially the same as the conventional carbon chemistry in young starless cores (e.g., Herbst 1983). Since the $\mathrm{C}^{+}+\mathrm{H}_{2}$ reaction is a slow radiativeassociation process, the efficiency to produce organic molecules is not very high.

(2) Warm carbon-chain chemistry $(T \sim 25 K)$ : When the temperature is raised up to $25 \mathrm{~K}, \mathrm{CH}_{4}$ can be evaporated. This realizes a temporary carbon-rich condition in the gas phase. Since $\mathrm{C}^{+}$already exists in stage $(1), \mathrm{CH}_{4}$ reacts with $\mathrm{C}^{+}$to produce carbon-chain molecules. For this process to be effective, the fractional abundance of $\mathrm{CH}_{4}$ relative to $\mathrm{H}_{2}$ in the gas phase should be higher than several times $10^{-7}$. In this case, $\mathrm{CH}_{4}$ can be a major reactant for $\mathrm{C}^{+}$in place of $\mathrm{OH}$ and other molecules. Note that $\mathrm{H}_{2} \mathrm{O}$ is still frozen on dust grains, and does not contribute to the $\mathrm{C}^{+}$destruction in this stage.

(3) Hot corino chemistry $(T=70-100 \mathrm{~K})$ : The ice mantle will be evaporated at this temperature. Complex organic molecules trapped in the ice mantle will also be released into the gas phase, which realizes the hot corino chemistry. At the same time, the $\mathrm{C}^{+}+\mathrm{CH}_{4}$ reaction will be suppressed, because overwhelming $\mathrm{H}_{2} \mathrm{O}$ molecules act as the main destructor of $\mathrm{C}^{+}$. The $\mathrm{C}^{+}+\mathrm{H}_{2} \mathrm{O}$ reaction just reproduces $\mathrm{CO}$ through $\mathrm{HCO}^{+}$. This would result in the deficiency of carbon-chain molecules.

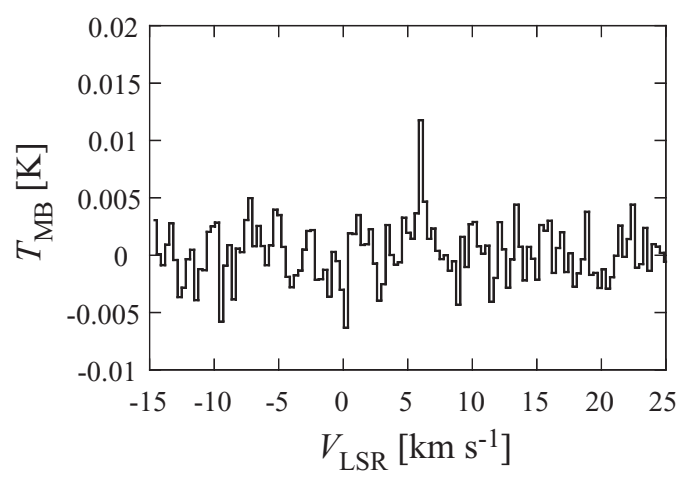

Figure 5. Spectral line profile of $\mathrm{CH}_{3} \mathrm{D}\left(J_{K}=1_{0}-0_{0}\right)$ observed toward L1527 with HHST. 
According to the above picture, the WCCC becomes effective only when the gas-phase $\mathrm{CH}_{4}$ abundance is higher than several times $10^{-7}$. In this case, the chemical evolution will proceed in order of (1), (2), and (3). If the $\mathrm{CH}_{4}$ abundance is not enough for WCCC, the evolution will proceed from (1) to (3) directly by skipping stage (2). Hence, the gas-phase $\mathrm{CH}_{4}$ abundance is a key factor for appearance of WCCC. In fact, we have recently detected the rotational spectral line of $\mathrm{CH}_{3} \mathrm{D}$ toward the prototypical WCCC source, L1527, and have found that the derived $\mathrm{CH}_{4}$ abundance $\left(f=(4.3-15.2) \times 10^{-6}\right)$ indeed fulfills this criterion (Figure 5). A significant spread in the $\mathrm{CH}_{4} / \mathrm{H}_{2} \mathrm{O}$ abundance ratio in the solid phase is seen in infrared observations of low-mass protostars (Öberg et al. 2008). Considering that the difference of the $\mathrm{CH}_{4}$ abundance would be amplified in abundances of carbon-chain molecules through their formation processes, it is highly likely that the abundance of $\mathrm{CH}_{4}$ supplied from grain mantles would control the chemical composition in the low-mass protostars. In the present stage, it is not certain whether the WCCC sources have a hot corino activity in the closest vicinity of the protostar. However, the hot corino activity should be weak even if it does exist, since the $\mathrm{CH}_{3} \mathrm{OH}$ lines $\left(J_{K}=7_{K}-6_{K}\right)$ are much weaker in the WCCC sources than in the hot corino sources (Jørgensen et al. 2007; Sakai et al. in prep) and large saturated COMs are not detected in L1527. This situation is schematically shown in Figure 6 .

From the above considerations, the chemical diversity would not originate from a simple evolutionary effect, but the different chemical compositions of grain mantles are needed to be considered. This is consistent with the fact that the hot corino sources are not always more evolved than the WCCC sources.

\section{hot corino sources}

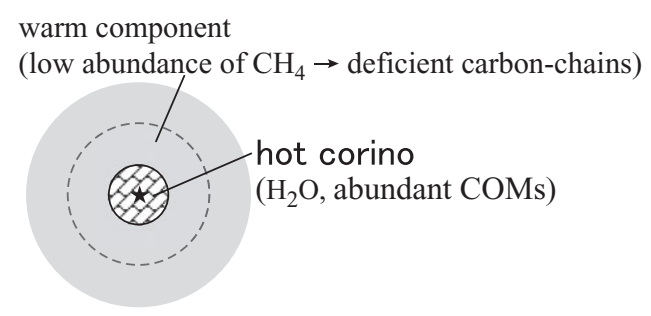

\section{WCCC sources}

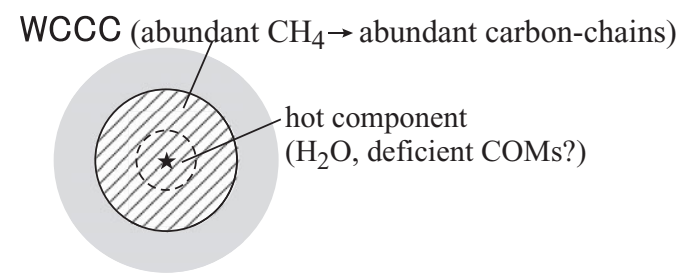

Figure 6. Physical appearance of the hot corino sources and the WCCC sources. In the hot corino sources, carbon-chain molecules are deficient over the protostellar core, whereas saturated COMs are abundant in the vicinity of the protostar (a few hundred AU). In the WCCC sources, carbon-chain molecules are distributed abundantly in a lukewarm region near the protostar (a few thousand $\mathrm{AU}$ ). On the other hand, large saturated COMs are not detected, indicating that the hot corino activity is very weak. 


\subsection{Origin of the Different Mantle Compositions}

As seen in the above unified picture of carbon chemistry in low-mass star forming regions, hot corino chemistry and the WCCC would reflect the different chemical compositions of grain mantles. This difference would reflect the past history during the starless core phase, when the grain mantle had grown up. One important factor is the duration time of the starless-core phase (Sakai et al. 2009a) (Figure 7). It could depend on large-scale environmental effects including magnetic field strength and turbulent energy input. The WCCC prefers the short starless-core phase. In this case, more carbon atoms are depleted directly onto dust grains before being converted to $\mathrm{CO}$ in the gas phase, which makes more $\mathrm{CH}_{4}$ on dust grains by hydrogenation. In contrast, the hot corino chemistry requires the long starless-core phase. In this case, CO is mainly depleted onto dust grains to form saturated $\mathrm{COMs}$, whereas $\mathrm{CH}_{4}$ is hardly produced.

The short starless-core phase for the WCCC sources is also supported by the deuterium fractionation ratios. Sakai et al. (2009b) observed many deuterated carbon-chain molecules, and found that the deuterium fractionation ratios are moderate $(2-7 \%)$. The $\mathrm{CH}_{2} \mathrm{DOH} / \mathrm{CH}_{3} \mathrm{OH}$ ratio is derived to be lower than $3 \%$, which is much lower than that found in hot corinos (Parise et al. 2006). The observed ratios mean less degree of the CO depletion, which is consistent with the short time scale of the starless-core phase.

The two cases shown in Figure 7 are the extreme ones, and the intermediate case would be possible, depending on the length of the starless-core phase. In this way, the continuous chemical variation shown in our $\mathrm{C}_{4} \mathrm{H}$ survey (Sakai et al. 2009a) can naturally be interpreted. If this is the case, we would be able to obtain novel information on the past contracting process of the parent core from what type of chemistry is now occurring near the protostar. We think that this can be a new approach to understanding physical processes in the early stages of star formation.

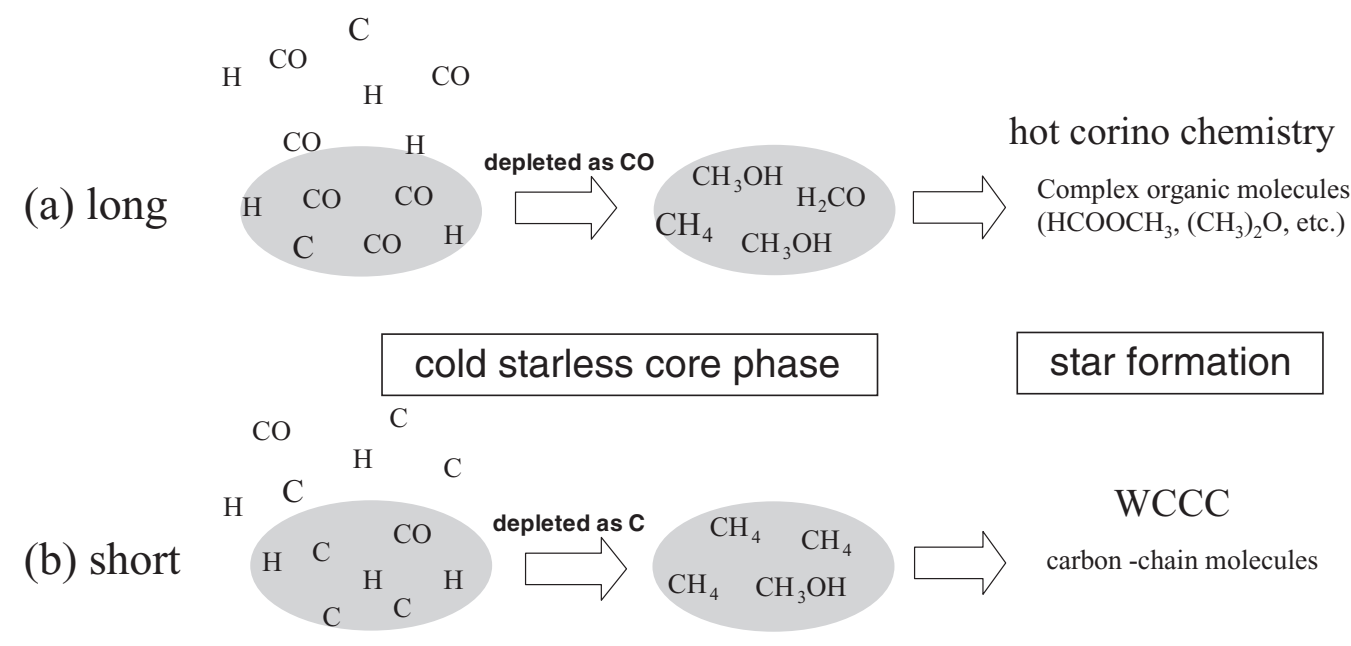

Figure 7. Origin of chemical variation. The two extreme cases are shown. The case (a) represents the chemical evolution for a long starless core phase. Most of the $\mathrm{C}$ atoms are converted to the $\mathrm{CO}$ molecules in the gas phase, which then deplete onto dust grains. They then form $\mathrm{CH}_{3} \mathrm{OH}, \mathrm{H}_{2} \mathrm{CO}$, and more complex organic molecules through hydrogenation reactions and radical reactions on the grain surfaces. On the other hand, the case (b) corresponds to a short starless-core phase in which a certain amount of the $\mathrm{C}$ atoms deplete directly onto the dust grains, and then form $\mathrm{CH}_{4}$ effectively through hydrogenation. This realizes WCCC. 


\section{Summary and Implications}

During the last decade, chemical compositions of low-mass star-forming regions have been investigated extensively, as summarized above. Now, we recognize the significant chemical diversity even among sources in a similar physical evolutionary stage. Nevertheless, we still have many unknowns on the chemical evolution of protostellar cores, some of which are highlighted below:

(1) We do not know whether the hot corino activity exists inside the WCCC sources, as mentioned above. According to the unified picture, the hot corino chemistry would occur in the vicinity of the protostar even for the WCCC sources. However, the hot corino activity is known to be weak in these sources. This means that saturated COMs are not well produced in the WCCC sources for some reasons. To address this problem, we have to study the weak hot corino activity in the WCCC sources with sensitive and high-resolution observations. Furthermore, it is necessary to understand the formation mechanism of saturated COMs on grain mantles in more detail.

(2) How unique are the WCCC and the hot corino chemistry among the low-mass star forming regions? We need to investigate chemical compositions of more low-mass protostellar cores for statistical arguments.

(3) How is the chemical diversity of the Class 0 sources brought into the later stages? Although the relation between hot corinos and protostellar disks is still unknown, saturated COMs are acturally distributed within a few 100 AU region around the protostar. On the other hand, carbon-chain molecules also exist in the closest vicinity of the protostar $(<500 \mathrm{AU})$ in the WCCC sources (Sakai et al. 2010). Thus, the chemical variation would be delivered to the protoplanetary disks. Its detailed processes are needed to be clarified.

Here we stress that the above questions are only major ones. We still have a big gap between chemistry of star-forming regions and that of protoplanetary disks. In the next decade, a dramatic progress is expected in this area with the full operation of ALMA.

Finally, we mention unique importance of COMs in tracing the chemical evolution. Observations of 'fundamental' molecules such as $\mathrm{CO}, \mathrm{HCN}$ and $\mathrm{HCO}^{+}$are essential to study the current physical structures and kinematics of star-forming regions. Their relatively high abundances ensure bright emissions in many sources to make such a business easy. However, fundamental species appear everywhere, and it is difficult to extract chemical characteristic of each source from their observations. On the other hand, COMs are much more sensitive to chemical and physical environments and are suitable for chemical characterization. A drawback is faintness of the lines, and hence, sensitive observations are necessary. If we can overcome this for instance with ALMA, complex molecules will gives us fruitful information, not "complex" information.

\section{Acknowledgements}

The authors are grateful to Yuri Aikawa, Michael Burton, Cecilia Ceccarelli, Eric Herbst, Tomoya Hirota, Jes Jørgensen, Nagayoshi Ohashi, Takeshi Sakai, and Yancy Shirley for their stimulating discussions. This study is supported by Grant-in-Aids from Ministry of Education, Culture, Sports, Science, and Technologies (21224002 and 21740135).

\section{References}

Agúndez., M. Cernicharo, J., Guélin, M., Gerin, M., McCarthy, M. C., \& Thaddeus, P. 2008, $A \& A, 478, \mathrm{~L} 19$

Aikawa, Y., Wakelam, V., Garrod, R. T., \& Herbst, E. 2008, ApJ, 674, 984 
Benson, P. J., Caselli, P., \& Myers, P. C. 1998, ApJ, 506, 743

Blake, G. A., Sutton, E. C., Masson, C. R., \& Phillips, T. G. 1987, ApJ, 315, 621

Blake, G. A., van Dischoeck, E. F., Jansen, D. J., Groesbeck, T. D., \& Mundy, L. G. 1994, ApJ, 428,680

Bottinelli, S., et al., 2004, ApJ, 615, 354

Bottinelli, S., et al., 2004, ApJ, 617, L69

Bottinelli, S., Ceccarelli, C., Williams, J. P., \& Lefloch, B. 2007, A\&A, 463, 601

Cazaux, S., et al., 2003, ApJ, 593, L51

Charnley, S. B., Tielens, A. G. G. M., \& Rodgers, S. D. 1997, ApJ, 482, L203

Collings, M. P., et al., 2004, MNRAS, 354, 1133

Cronin, J. R. \& Pizzarello, S. 1997, Sci., 275, 951

Garrod, R. T. \& Herbst, E. 2006, A\&SA, 457, 927

Harada, N. \& Herbst, E. 2008, ApJ, 685, 272

Hassel, G. E., Herbst, E., \& Garrod, R. T. 2008, ApJ, 681, 1385

Herbst, E. 1983, ApJS, 53, 41

Herbst, E. \& van Dishoeck, E. F. 2009, ARAA, 47, 427

Jørgensen, J. K., Schöier, F. L., \& van Dishoeck, E. F. 2002, A\&̈A, 389, 908

Jørgensen, J. K., Bourke, T. L., Myers, P. C., Schöier, F. L., van Dishoeck, E. F., \& Wilner, D. J. 2005, ApJ, 632, 973

Jørgensen, J. K., et al., 2007, ApJ, 659, 479

Kaifu, N., et al., 2004, PASJ, 56, 69

Kuan, Y.-J., et al., 2004, ApJ, 616, L27

Millar, T. J., Herbst, E., \& Charnley, S. B. 1991, ApJ, 369, 147

Nomura, H. \& Millar, T. J. 2004, A\& $A, 414,409$

Öberg, K. 2008, ApJ, 678, 1032

Parise, B., et al., 2006, A\&SA, 453, 949

Sakai, N., Sakai, T., \& Yamamoto, S. 2006, PASJ, 58, L15

Sakai, N., Sakai, T., Osamura, Y., \& Yamamoto, S. 2007, ApJ, 667, L65

Sakai, N., Sakai, T., Hirota, T., \& Yamamoto, S. 2008a, ApJ, 672, 371

Sakai, N., Sakai, T., \& Yamamoto, S. 2008b, ApJ, 673, L71

Sakai, N., Sakai, T., \& Yamamoto, S. 2008c, ApधSSS, 313, 153

Sakai, N., Sakai, T., Hirota, T., Burton, M., \& Yamamoto, S. 2009a, ApJ, 697, 769

Sakai, N., Sakai, T., Hirota, T., \& Yamamoto, S. 2009b, ApJ, 702, 1025

Sakai, N., Sakai, T., Hirota, T., \& Yamamoto, S. 2010, ApJ, 722, 1633

Suzuki, H., et al., 1992, ApJ, 392, 551

van Dishoeck, E. F., Blake, G. A., Jansen, D. J., \& Groesbeck, T. D. 1995, ApJ, 447, 760

Watanabe, N. \& Kouchi, A. 2002, ApJ, 571, L73

\section{Discussion}

Doug Johnstone: You suggest that the difference between Hot Corino Sources and WCCC sources is due to the speed with which they collapse but how important might the environment of the core be? For exampe, a core at the edge of a cloud may live in a higher radiation field?

NAMI SAKAI: I agree that environmental effects are important. We should concretely identify what types of environmental effects discriminate between the hot corino chemistry and WCCC. One important factor would be the large scale star-formation activity in the region where the protostar resides. Although the number of the sample is limited, a region harboring hot corinos is usually a formation site of star clusters, whereas a region harboring WCCC sources is relatively quiescent. Such a difference in the large-scale environment may affect the contracting speed of the clouds. The position of the cores in a parent cloud could also be a possible cause, as you mention. More statistics are essential. 
E. F. VAN Dishoeck: It is not clear to me why you so easily dismiss the information on the "spread" in observed $\mathrm{CH}_{4}$ ice abundances toward pre-stellar cores and protostars, since these data should probe close to the sublimation temperature of $\mathrm{CH}_{4}$ ice.

NAMI SAKAI: We think that the $\mathrm{CH}_{4}$ ice abundance would strongly depend on the density distribution and the temperature structure, and hence, we have directly measured the gas-phase $\mathrm{CH}_{4}$ abundance in a warm region to test our WCCC scenario. However, I agree that we should more carefully make use of the $\mathrm{CH}_{4}$ ice abundances reported so far, because a factor of difference in the $\mathrm{CH}_{4}$ abundance would cause a big difference in the abundances of complex species.

MARTin Cordiner: Are there any other observational tracers to test the idea a rapid freeze-out produces elevated ice $\mathrm{CH}_{4}$ abundances?

NAmi SAKAI: The molecular D/H ratios would be an independent tracer. The relatively low fractions observed in L1527 suggest the freeze-out of gaseous species in early stages of chemical evolution of its parent cloud.

S. Viti: TPD experiments (e.g. Collings et al. 2004, Viti et al. 2004) show that not all the $\mathrm{CO}$ and $\mathrm{CH}_{4}$ evaporate at $20 \mathrm{~K}$ and $25 \mathrm{~K}$ respectively. In fact you should have about $40 \%$ of $\mathrm{CH}_{4}$ still on the grains up to $\sim 70 \mathrm{~K}$. How does this agree your conclusion?

NAmi SAKAI: Thank you very much for your comments. It would not affect seriously on our conclusion. Even if that portion $(\sim 40 \%)$ of the $\mathrm{CH}_{4}$ ice still remains on grains and is released into the gas phase in the hot $(>70 \mathrm{~K})$ region, carbon-chain production would not be effective, because $\mathrm{H}_{2} \mathrm{O}$ becomes the main destructor of $\mathrm{C}^{+}$in such a hot region. However, your point will be important for detailed modeling. 